INRA Prod. Anim.,

2012,25 (2), 159-168

\title{
Hommes et animaux d'élevage au travail : vers une approche pluridisciplinaire des pratiques relationnelles
}

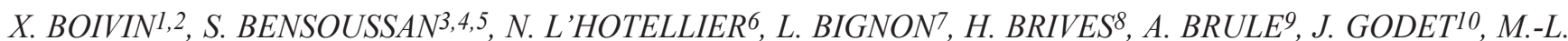
GRANNEC ${ }^{11}$, M. HAUSBERGER ${ }^{12}$, F. KLING-EVEILLARD ${ }^{13}$, C. TALLET $T^{4,5}$, V. COURBOULAY3

${ }^{1}$ INRA, UMR1213 Herbivores, F-63122 Saint-Genès-Champanelle, France

${ }^{2}$ Clermont Université, VetAgro Sup, UMR Herbivores, BP 10448, F-63000 Clermont-Ferrand, France

${ }^{3}$ IFIP, Institut du Porc, La Motte au Vicomte, BP 3, F-35651 Le Rheu, France

${ }^{4}$ INRA, UMR1348 Physiologie, Environnement et Génétique pour l'Animal et les Systèmes d'Elevage, F-35590 Saint-Gilles, France

${ }^{5}$ Agrocampus Ouest, UMR Physiologie, Environnement et Génétique pour l'Animal et les Systèmes d'Elevage, F-35590 Saint-Gilles, France

${ }^{6}$ CCMSA, Les Mercuriales - Tour Ponant, 40 rue Jean Jaurès, F-93547 Bagnolet, France

7 ITAVI, UMT BIRD, Centre de Tours, F-37380 Nouzilly, France

8 AgroParisTech, UFR Sociologie, 16 rue Claude Bernard, F-75231 Paris, France

${ }^{9}$ Institut de l'Elevage, Monvoisin, F-35652 Le Rheu, France

10 ISARA-Lyon, 23 rue Jean Baldassini, F-69364 Lyon, France

${ }^{11}$ Chambre Régionale d'Agriculture de Bretagne, pôle régional de recherche porcs-aviculture, F-35042 Rennes, France

12 Université de Rennes 1, UMR Ethologie Animale et Humaine, campus de Beaulieu, 263 Аvenue du General Leclerc, F-35042 Rennes, France

${ }^{13}$ Institut de l'Elevage, SIM, 149 rue de Bercy, F-75595 Paris, France Courriel :xavier.boivin@clermont.inra.fr

Autant pour des enjeux d'efficacité du travail, de confort et de sécurité au travail, de satisfaction professionnelle pour les personnes qui travaillent avec des animaux d'élevage, qu'en termes de bien-être animal, il est essentiel de bien maitriser les facteurs d'une relation homme-animal qui bénéficie aux deux. Cette maitrise passe par une démarche pluridisciplinaire associant les sciences sociales et l'éthologie afin de prendre en compte les points de vue de l'homme et de l'animal.

Le métier d'éleveur est par définition centré sur l'animal. L'éleveur prend en compte les besoins physiologiques et psychologiques de l'animal afin qu'il produise dans les meilleures conditions. Dans son travail avec l'animal, il cherche ainsi à associer rentabilité économique et bien-être animal (KlingEveillard et al 2009). D'un point de vue réglementaire, l'animal est aujourd'hui reconnu comme "être sensible». (Article L214-1 du Code Rural ${ }^{1}$ ). Une proposition de loi du sénat lors de la session 2010/2011 définit juridiquement ce statut d'être sensible : «Ainsi, l'aptitude à ressentir la douleur et à éprouver d'autres émotions, au regard, entre au-tres, de l'effectivité de leur système nerveux supérieur, semble apparaître comme l'une des bases indispensables à une telle définition ${ }^{2} »$. Aussi, l'animal doit être placé par son propriétaire «dans des conditions compatibles avec les impératifs biologiques de son espèce». Il est nécessaire de «leur éviter [aux animaux] des souffrances lors des manipulations inhérentes aux diverses techniques d'élevage, de parcage, de transport et d'abattage» (articles 214-1 et 214-3 du Code Rural ${ }^{3}$ ).

L'animal n'est donc pas passif par rapport à l'activité d'élevage. Ses perceptions de la situation et de l'éleveur sont des facteurs clés de son comportement et de son adaptation aux systèmes d'élevage (Boivin et al 2003). L'évaluation du bien-être de l'animal intègre la qualité de la relation entre

\footnotetext{
1 http://admi.net/code/CRURALNL-L214-1.html

2 http://www.senat.fr/leg/ppl10-575.html

3 http://admi.net/code/CRURALNL-L214-3.html
} 
l'homme et l'animal en prenant en compte la réaction des animaux à l'homme (Veissier et al 2010). Le temps et la qualité du travail réalisés au contact direct ou indirect de l'animal comme notamment la surveillance et les soins, sont reconnus comme primordiaux dans la construction de cette relation (Boivin et al 2007, Dockes et Kling-Eveillard 2007). Mais l'éleveur fait face aujourd'hui à de multiples contraintes (respect des différentes normes sanitaires, environnementales, de bien-être, des cahiers des charges de labellisation ou de certification, adaptabilité maximum requise suivant les variations de l'environnement économique et climatique, besoin de formation aux nouveaux outils...). Bien gérer son temps, comme pour beaucoup d'autres professions, est devenu un enjeu important pour concilier investissement professionnel et choix de vie personnels (vacances, autres activités). Tous ces facteurs peuvent générer une altération des rapports et des contacts entre l'homme et l'animal. De nombreuses disciplines se sont intéressées à la relation entre l'homme et l'animal, mais le plus souvent en ne prenant en compte que le point de vue de l'animal ou au contraire que celui de l'homme. Un zootechnicien considère la relation hommeanimal dans sa logique de production. Un sociologue, ou un ergonome la voit surtout du point de vue de l'humain dans sa façon de réfléchir, de se comporter et de s'organiser face à l'animal, dans un contexte social varié (famille, conseillers, vétérinaires...). De son côté, un éthologue envisage généralement la question de la relation hommeanimal du point de vue de l'animal (comment l'animal perçoit son partenaire humain?).

Dans le cadre du projet «Evaluation du bien-être animal ${ }^{4}$ » et du Réseau Mixte Technologique intitulé «Bienêtre animal et systèmes d'élevage», un groupe réunissant éthologues, zootechniciens, sociologues et ergonomes travaillant sur différentes espèces (ruminants, porcs, chevaux, oiseaux, lapins) a été constitué afin de confronter leurs points de vue et leur compréhension de la relation homme-animal, notamment sur la description et la conceptualisation de cette dernière, son évaluation et les moyens de l'améliorer. L'objectif final du travail est de modifier les formations existantes des éleveurs en y amenant une nouvelle réflexion sur leurs pratiques dans la construction de la relation avec leurs animaux. L'article présenté ici est une synthèse des premiers travaux menés dans ce sens par le groupe. Ce dernier a limité son champ de réflexion aux animaux d'élevage dans un contexte de travail et s'est attaché à explorer et à prendre en compte le point de vue de chacun des acteurs de la relation : l'homme et l'animal.

\section{1 / La relation homme- animal vue du côté de l'homme}

Les travaux récents s'intéressant à la relation homme-animal d'élevage du point de vue de l'homme sont au croisement de différentes disciplines telles que la zootechnie et la sociologie du travail (Porcher 2001, Dockes et KlingEveillard 2007, Fiorelli 2010). Ces travaux considèrent le travail de l'éleveur dans son ensemble. Ils tiennent compte de son environnement, de l'influence des facteurs internes (état émotionnel...) et externes (pression sociale...), de sa façon de construire son identité au travail ainsi que des perceptions et des points de vue qu'il partage avec ses collègues (Dufour et Dedieu 2010). Ces travaux ont permis de prendre du recul par rapport aux choix individuels des exploitants, de comprendre leurs motivations et leurs façons d'agir, notamment par rapport à l'animal et leur métier. Ils permettent aussi d'aborder la question de l'organisation du travail. De ce point de vue, cette approche rejoint celle dite systémique de l'ergonomie du travail (Darses et de Montmollin 2006). L'approche systémique étudie la compréhension du travail et l'organisation du travail en intégrant la conception des bâtiments et des équipements ainsi que les interactions entre l'homme et son environnement. Le travailleur est vu ici comme acteur dans le système. L'intervention ergonomique peut se faire à plusieurs niveaux : d'un point de vue global (qu'est-ce qui est pénible sur cette exploitation ?) ou ciblé (comment améliorer tel poste de travail ?). Dans les systèmes d'élevage, l'intervention ergonomique porte le plus souvent sur ce dernier niveau. En effet, c'est celui qui paraît être pour les éleveurs le plus accessible du fait des améliorations tangibles et immédiates qu'il peut apporter (modification spatiale d'un poste de travail par exemple ou posture de travail adaptée à la contention des animaux), (Montméas et al 2006). Mais il peut être nécessaire d'apporter des modifications plus profondes dans l'organisation du travail en modifiant l'attribution des postes et des tâches des travailleurs, voire même des pratiques de travail. Sans en arriver systématiquement à des transformations, une prise en compte globale de ce qui se joue dans l'entrepri- se peut apporter un éclairage sur les facteurs externes perturbateurs de la relation homme-animal. Par exemple, un manque de personnel ou des difficultés économiques peuvent générer une augmentation du stress de l'éleveur qui peut à son tour favoriser l'apparition de comportements «agressifs» chez l'animal (L'Hotellier et al 2007). Mais les travaux sur ce thème sont encore très peu nombreux.

Dans la suite de cette partie, nous aborderons d'abord la question de la relation homme-animal par l'analyse du temps de travail de l'éleveur au contact de l'animal. Puis, nous nous intéresserons au fonctionnement de l'éleveur face à ses animaux.

\section{1 / Temps de travail et contacts avec l'animal}

La question du temps que le professionnel se donne pour avoir une «bonne relation» avec son animal ou son troupeau est toujours évoquée lors des sessions de formation sur la relation homme-animal et la manipulation/ contention (X. Boivin, communication personnelle). Pour les éleveurs, cette «bonne relation» se caractérise par le fait d'avoir des animaux qui ne montrent pas de réaction de peur face à l'homme et qui permettent au manipulateur de travailler efficacement et en toute sécurité. Les éleveurs de bovins limousins notamment, mettent principalement en avant la présence de l'éleveur et son comportement comme les deux composantes les plus importantes dans la construction de la relation hommeanimal (Boivin et al 2007). Pourtant, nombre d'entre eux disent qu'ils ne disposent plus aujourd'hui de suffisamment de temps pour construire cette relation. Beaucoup pointent l'accroissement du volume global de travail et en particulier du travail administratif, le temps passé à s'informer, se former, commercialiser, etc. Souvent ils évoquent le sujet des vacances, de la vie personnelle à côté de la vie professionnelle et font un parallèle entre leurs activités et les autres catégories sociales travaillant majoritairement $35 \mathrm{~h}$ par semaine. Dans ces conditions, qu'en est-il réellement du temps passé auprès des animaux et du temps alloué à construire la relation avec ces derniers ?

Afin de pouvoir évaluer le temps passé auprès des animaux, une quantification de ce temps s'avère intéressante mais n'a jamais été en elle-même un objet d'étude. Il est souvent difficile méthodologiquement de le quantifier sans s'immerger dans l'exploitation

\footnotetext{
${ }^{4}$ Projet «Evaluation du Bien-être animal», CASDAR N9111
} 
comme pourraient le faire les anthropologues (Metz 1986). On conçoit aisément la lourdeur du dispositif d'observation, le risque de perturbations liées à la présence de l'observateur et la difficulté d'étudier la variabilité existante entre exploitations. Il est cependant possible d'en faire une première approximation par l'évaluation des temps de travail en élevage sur la base d'enquêtes réalisées à partir de la méthode Bilan Travail (Dedieu et Servière 1999). Cette dernière distingue deux catégories de travailleurs: ceux de la «cellule de base» (travailleurs permanents) et les autres travailleurs (salariés, bénévoles, aide). Trois grands types de travaux sont identifiés et quantifiés en nombre d'heures par jour : le travail d'astreinte, le travail de saison (manipulations de troupeaux, interventions sur les surfaces) et le travail rendu (remboursement de l'entraide perçue entre exploitations). Parmi ces différents types de travail, il apparaît intéressant de regarder plus particulièrement le travail d'astreinte qui correspond aux soins journaliers apportés aux animaux. Par définition il s'effectue quotidiennement, il est difficile à concentrer et surtout ne peut pas être différé (Dedieu et Servière 1999). En ce sens, il implique de passer du temps auprès des animaux, d'être en contact physiquement ou par la simple présence dans le bâtiment. Selon le type d'élevage, les travaux d'astreinte peuvent comprendre l'enlèvement des animaux, la reproduction, leur alimentation, la traite, la gestion du pâturage, la surveillance, le paillage, le raclage ou le nettoyage des bâtiments, etc. (Gaudillère 2009). Il peut également être pertinent de prendre en compte le travail de saison. En effet, celui-ci comprend des manipulations ponctuelles avec les troupeaux (tonte, prophylaxie, déplacements...) qui peuvent avoir un impact important sur la relation homme-animal mais qui ne sont malheureusement actuellement pas aussi finement quantifiées (en jours et non en heures).

Des études s'appuyant sur une quantification du temps de travail montrent que les temps de présence auprès des animaux varient fortement d'une espèce à l'autre (Procoli 2004, Cournut et Chauvat 2012). Ceci est lié notamment à la filière concernée et au sentiment d'appropriation de l'animal par l'éleveur ainsi qu'à l'intensification de l'élevage (notamment le nombre d'animaux par travailleur). En production porcine, la majorité des tâches sont incluses dans le travail d'astreinte (Grannec 2010). En effet, l'organisation du travail est dictée par la conduite en bandes du troupeau qui ne permet pas ou peu de différer la réalisation de la plupart des tâches. La description du temps de travail peut se faire de façon plus précise en tenant compte du cycle de vie des animaux. Par exemple chez les naisseurs en production porcine, l'organisation du travail en cycles (saillie, mise-bas, sevrage) les amène à être très présents aux moments clés du cycle de reproduction, notamment en maternité à la mise-bas et à la période des inséminations, mais les visites sont plus espacées pendant la gestation (Grannec 2010). En production ovine, on observe une grande variabilité entre les systèmes, certains occasionnant du gardiennage et donc une forte présence de l'homme (Cournut et Chauvat 2012). Les systèmes qui exigent le plus de travail d'astreinte par personne sont ceux dans lesquels le fonctionnement de l'exploitation repose sur une seule personne ou quand le gardiennage se fait sur de longues périodes (en montagne par exemple).

Les élevages laitiers (caprins, bovins, ovins) sont les systèmes d'élevage où le temps passé et le nombre de contacts avec l'animal par les professionnels sont les plus importants (Cournut et Chauvat 2012). Ces élevages nécessitent des soins que l'on retrouve dans d'autres filières (alimentation, soins vétérinaires, surveillance) mais exigent en plus de traire les animaux une à deux, voire trois fois par jour. L'astreinte de la traite conduit à des contacts supplémentaires plus longs et directs (contacts, paroles) par rapport aux autres productions. Le temps d'astreinte semble beaucoup plus faible dans les filières avicoles bien qu'il y ait à notre connaissance peu d'études sur le sujet et dans les élevages de bovins allaitants (Cournut et Chauvat 2012). Bien que la surveillance soit un enjeu important, le travail en bandes diminue le temps que le professionnel passe avec chaque animal. Par exemple, il a été évalué qu'une conduite individuelle de lapines de reproduction nécessite quatre fois plus de temps d'astreinte qu'une conduite en bande unique (Anonyme 2006). Le calcul du temps passé par l'éleveur au contact de chaque animal est difficile à interpréter du point de vue de la relation homme-animal compte tenu de la variabilité de configuration des bâtiments, et suivant si les animaux sont en élevage collectif ou en cage individuelle, voire les deux. Ces différents paramètres vont influencer la possibilité de contacts directs ou indirects, et donc l'exposition de l'animal à l'homme (Chauvat et Servière 2005).

L'analyse du temps de travail passé par l'éleveur à proximité ou au contact de ses animaux montre donc une grande diversité selon les filières. Elle montre aussi une grande variabilité entre les éleveurs eux-mêmes au sein d'une même filière (Cournut et Chauvat 2012).
Cette analyse permet de s'interroger sur la diversité des profils individuels des éleveurs. Elle permet aussi de s'interroger sur leurs logiques d'élevage c'est-àdire sur la façon dont ils prennent en compte l'ensemble des buts qu'ils souhaitent atteindre, les règles techniques et professionnelles auxquelles ils sont soumis et les contextes institutionnels, économiques et sociaux incertains dans lesquels ils évoluent (Le Guen 2006). Les choix et les logiques d'éleveurs conditionnent forcément la relation homme-animal par des variations de temps de présence et de comportement envers l'animal. Par exemple, les solutions d'automatisation, de stabulation libre diminuent le travail d'astreinte en réduisant la pénibilité du travail et en facilitant son organisation. Mais Lamine (2006) estime que cette automatisation mène à une forme d'artificialisation du vivant. Ces solutions d'aide au travail modifient le rapport des éleveurs aux animaux. Les éleveurs passent plus de temps à les surveiller, mais moins à leur contact physique, ce qui dessine les principales différences de proximité entre éleveurs et animaux. Certains travaux soulignent le sentiment des éleveurs de manquer de temps, la pression exercée par les rythmes de travail trop élevés (Porcher 1997). Lamine (2006) évoque quant à elle les éleveurs qui souhaitent «prendre du temps pour bien faire». Les éleveurs sont tiraillés entre le temps de l'urgence (production et adaptation aux législations), le temps qu'ils se donnent pour être avec leurs animaux ainsi que le temps d'apprentissage de leur métier et de leurs bêtes.

\section{2 / Profils d'éleveurs et logiques d'élevage face à l'ani- mal}

D’une façon générale, certaines dimensions de l'homme telles que la personnalité (introverti/extraverti, confiance en soi), l'humeur (irritabilité, agressivité, calme...) ou l'empathie (faculté intuitive de se mettre à la place d'autrui, de ressentir...) peuvent affecter ses comportements envers les animaux (Seabrook 1972, English 1991). Un manipulateur agité, nerveux ou manquant de confiance en lui est beaucoup moins efficace avec les bovins en provoquant plus souvent des réactions de peur ou d'agression chez les animaux qu'un manipulateur calme, expérimenté et sûr de lui (Renger 1975). Ces réactions de l'animal influencent en retour les représentations que l'homme peut avoir des animaux, notamment ses représentations comportementales (c'est-à-dire les croyances sur l'effet de son propre comportement sur la réaction de l'animal). La théorie psychologique de l'action raisonnée de Fishbein (1980) montre que ces représentations sont des bons 
prédicteurs des habitudes comportementales. Cette théorie, appliquée dans le cadre de la relation homme-animal d'élevage, d'abord par G. Coleman et P. Hemsworth, puis par différents autres auteurs, a été très utile pour comprendre une partie de la variabilité des comportements humains entre fermes ou abattoirs et a été prise en compte dans la formation des éleveurs (Hemsworth et Boivin 2011). Les représentations que les éleveurs ont de leurs animaux influencent également le choix de leur système d'élevage.

Pour Lamine (2006), deux statuts de l'animal coexistent pour les éleveurs : l'animal être vivant et domestique en relation avec les humains et l'animal futur aliment. Chez un certain nombre d'éleveurs, elle constate que la relation aux animaux prime, que l'animal suscite de l'affection, de la compassion et interagit avec l'homme. Les éleveurs se sentent responsables de leurs animaux et sont ainsi toujours affectés par les accidents sanitaires de leur troupeau (Dockes et Kling-Eveillard 2007). Mais cette dimension affective, empathique ne doit pas masquer une autre réalité que Lamine (2006) observe. Pour l'éleveur, l'animal est avant tout un «compagnon» de travail. La relation qui s'établit est surtout une relation professionnelle (Dockes et Kling-Eveillard 2007). A cela s'ajoute le fait que le métier d'éleveur et les moyens de production sont souvent hérités (surtout en production bovine et porcine). On observe donc souvent une relation d'ordre patrimoniale avec l'animal dont il ne faut pas négliger le caractère fortement contraignant. Dans ce contexte, l'éleveur n'a pas eu le choix de l'espèce ou de la race élevée et se retrouve, du moins au départ, à pérenniser des pratiques avec lesquelles il n'est pas forcément d'accord. Dès lors, on voit bien que le choix de l'espèce animale se combine à des choix économiques et des critères personnels de goût (KlingEveillard et al 2009). Dans ce cadre de la relation de travail avec l'animal, une typologie d'attitudes d'éleveurs a pu être faite par Dockes et Kling-Eveillard (2007). Quatre attitudes sont décrites : l'éleveur pour l'animal (l'éleveur a une relation affective avec ses animaux, il aime le contact avec eux), l'éleveur avec l'animal (l'éleveur s'occupe de l'animal comme d'un être sensible, avec qui on communique sans affectivité), l'éleveur malgré l'animal (la relation homme-animal est instrumentalisée, l'éleveur limite le contact avec l'animal) et l'éleveur pour la technique (la relation homme-animal n'est pas essentielle dans leur métier, l'animal a seulement des besoins physiologiques). Ces attitudes se répartissent sur deux axes que les auteurs représentent de façon orthogonale : la proximité avec l'animal d'une part et la passion pour la technique d'autre part. Au travers des entretiens, les auteurs recensent différentes visions de l'animal chez les éleveurs :

- l'animal instrumentalisé : fonctions de production et besoins physiologiques (cas des éleveurs malgré l'animal et pour la technique),

- l'animal communiquant : développement de relations d'échanges (cas des éleveurs avec l'animal),

- l'animal affectif : relation d'attachement avec l'homme à double sens (cas des éleveurs pour l'animal), rejoignant le concept d'intersubjectivité entre l'homme et l'animal (Porcher 2001).

Dockes et Kling-Eveillard (2007) s'interrogent sur cette diversité et surtout sur la relation qui pourrait exister entre ces représentations de leur métier par les éleveurs, leurs pratiques vis-àvis de l'animal et les conséquences sur la relation homme-animal et sur le bienêtre animal.

Cette composante relationnelle n'est qu'une des rationalités qui détermine le rapport subjectif des éleveurs au travail (Fiorelli 2010). Les autres composantes sont d'ordre économique, technique, identitaire mais aussi en lien avec l'engagement de son corps dans le travail (dehors/dedans, rythme...) (Fiorelli et al 2012 ce numéro). Le dimensionnement des activités (activité agricole, non agricole, non activité), la gestion du temps, la gestion du collectif de travail (composition et répartition des tâches entre les membres du collectif), les modalités d'élevage (choix de conduite du troupeau et des surfaces), et les bâtiments et équipements sont autant d'éléments qui vont, suivant les éleveurs, composer l'organisation du travail en y jouant un rôle de pivot (élément incontournable et structurant) ou de levier (élément ajustable et modifiable de manière à ce que l'éleveur organise son travail de manière satisfaisante).

Dans l'élevage, construire une relation avec l'animal demande de savoir faire des choix dans la gestion de son temps et donc dans son organisation du travail. On peut se demander pour chaque éleveur si construire une bonne relation homme-animal est pour lui une activité pivot ou une activité de levier. De même, on peut s'interroger sur le temps imparti ainsi que sur la qualité du travail dévolue à chaque tâche et qui peut affecter la relation homme-animal dans un contexte où le choix des éleveurs est de combiner l'ambition professionnelle et l'aspiration à un mode de vie.

\section{2 / La relation homme- animal en élevage du point de vue de l'animal}

\section{1 / L'éthologie animale : un cadre de référence dans la rela- tion homme-animal}

L'éthologie est une science qui étudie le comportement dans toutes ses manifestations et à tous les niveaux d'organisation : de la cellule à l'organisme entier et aux sociétés animales. L'éthologie étudie non seulement les causes qui déterminent les comportements mais aussi leurs fonctions (Campan et Scapini 2002). Le comportement peut se définir comme un ensemble de manifestations motrices observables, organisées et coordonnées. C'est une science systémique qui analyse le comportement de l'animal dans son environnement. Une des dimensions essentielles de cet environnement est la présence d'autres individus, qu'ils soient de la même espèce ou d'une espèce différente. L'éthologie cherche donc à comprendre le point de vue de l'animal, notamment dans sa dimension perceptuelle le «Umwelt». Ce concept élaboré à la fin du XIXème siècle (von Uexküll 1864-1944, cité par Campan et Scapini 2002) montre que chaque espèce animale vit dans un univers qu'il perçoit de façon différente des autres espèces, et donc différente de nous, l'espèce humaine. Elle prend également en compte l'histoire de l'espèce (phylogenèse) et de l'individu (ontogenèse).

L'éthologie propose un cadre conceptuel de la relation homme-animal fondé sur les interactions homme-animal (Estep et Hetts 1992). Les interactions entre l'animal et l'homme se succèdent de la même façon qu'elles se succèdent entre congénères. L'animal ne fait pas que réagir à l'homme et à son comportement, il interagit très fréquemment dès sa naissance avec celui-ci qui le soigne, le nourrit, le surveille, le manipule et l'utilise à des fins de production. C'est l'ensemble de ces interactions qui module la perception qu'a l'animal de l'homme (soigneurs, vétérinaire, inséminateurs...) et réciproquement, et qui permet de construire la relation entre les individus. En effet, chacun des partenaires de cette relation identifie et adapte en conséquence son comportement à l'autre, voire aux autres par discrimination ou généralisation ; il a une mémoire des interactions. Dès lors, que ce soit pour les partenaires de ces interactions répétées mais aussi pour un observateur extérieur qui les suit sur la durée il apparaît possible de prévoir l'issue des futures interactions. 
Pour étudier cette relation, l'éthologie propose d'examiner la perception (sensations, évaluation de la situation et émotions, représentations) de chacun des partenaires par l'autre en observant concrètement leur réaction à cet autre (Waiblinger et al 2006, Hemsworth et Boivin 2011) lors de situations homogènes tout en prenant en considération le fait que ces réactions peuvent être affectées par des facteurs extérieurs (environnement physique ou social) ou par des états internes variables tels que la faim ou l'humeur de chaque protagoniste.

\section{2 / Des outils d'évaluation de la réaction des animaux à l'homme}

De très nombreuses études depuis 40 ans ont cherché à évaluer la relation homme-animal du point de vue de l'animal. En 2006, un groupe d'éthologues expérimentés dans le domaine des animaux d'élevage s'est réuni pour analyser de façon critique les différents tests utilisés dans la littérature pour évaluer la réaction des animaux à l'homme, soit en expérimentation, soit en ferme (Waiblinger et al 2006). Trois types de tests ressortent de cette recherche :

- des tests de réaction à un homme passif (distance, latence d'approche, temps de contact...). L'homme passif est considéré comme «standard» car son comportement ne peut en théorie affecter les réactions de l'animal («the human standard approach test»). Développé par P. Hemsworth avec les porcs et utilisé depuis chez de nombreuses espèces, ce test a bien montré sa capacité à distinguer les réponses des animaux dont l'histoire avec l'homme était positive (répétition d'interactions renforçatrices du contact homme-animal) ou négative (répétitions d'interactions aversives, Hemsworth et al 1986, 1987, 1990). Un porc interagit facilement avec un homme passif mais de moins en moins s'il apprend au cours de sa vie que l'homme est aversif.

- des tests où la personne est en mouvement ou introduit sa main dans la cage (distance de fuite, nombre d'animaux autour de l'homme, nombre d'animaux touchés...). Le comportement de l'homme reste simple (marcher à allure régulière, toucher l'animal sur le dos...) pour être facile à standardiser et pour distinguer au mieux la seule réponse de l'animal (Jones 1993, Boivin et al 2009, Bokkers et al 2009). Ces tests sont un bon outil d'évaluation du bienêtre animal, et ont d'ailleurs été repris et adaptés dans le projet "Welfare Quality $(\mathrm{R})$ dont l'objectif était de mettre au point une méthode d'évaluation du bien-être animal en ferme. Dans ce projet, il a par exemple été mis en évidence que la distance à laquelle on peut appro- cher des vaches laitières en stabulation libre ou aux cornadis peut être mise en relation avec le comportement des vachers pendant la traite. Il a été montré que la distance de fuite moyenne des animaux dans une ferme est statistiquement corrélée avec la proportion de contacts positifs (caresses, paroles calmes...) par rapport aux contacts négatifs (coups/cris...) que les hommes leur donnent (Hemsworth et al 2000, Waiblinger et al 2003). On retrouve des relations identiques en élevage de veaux de boucherie (Lensink et al 2000), en élevage de porc (Hemsworth et al 1989 , $1994 \mathrm{~b})$ et de poulets de chair (Hemsworth et al 1994a).

- des tests d'évaluation des réactions aux manipulations lors de déplacement d'animaux, de capture, de contention. Contrairement aux deux autres tests où le comportement de l'homme peut être reproduit quasiment à l'identique, le fait de déplacer l'animal oblige l'homme à tenir compte des réactions de ce dernier. Néanmoins on considère que l'effet du manipulateur sur cette réaction peut être atténué par l'entraînement, ou bien qu'il est négligeable au regard de la prédominance de la variabilité animale (en fonction de l'origine génétique ou de l'histoire des animaux) (Boivin et al 1992).

En éthologie appliquée, ces observations standardisées et quantitatives des réactions des animaux face à l'homme (fréquence, durée...) servent de base à l'évaluation soit du comportement de l'animal, soit du bien-être de l'animal au sein de la ferme, voire du comportement de l'éleveur vis-à-vis de ses animaux si l'on peut avoir accès à un échantillon d'animaux représentatifs de l'élevage. Cependant dans une ferme, l'environnement est variable. La mise au point des protocoles d'évaluation et la validité des mesures réalisées dépendent donc beaucoup de la rigueur avec laquelle est suivi le protocole avant et pendant les mesures (Waiblinger et al 2006). Les observateurs doivent être formés pour avoir en tête les sources possibles de biais et les éliminer au maximum. Si celles-ci ne peuvent pas être éliminées lors des phases de prétest et durant le test, les observateurs doivent être en mesure de les contrôler au maximum. L'identité, l'aspect et le comportement des observateurs et/ou des manipulateurs sont des éléments très importants dans la variation des comportements observés face à l'homme. De nombreuses études sur différentes espèces (porcs, moutons, bovins, chevaux, volailles...) montrent que les animaux peuvent faire la différence entre différentes personnes et dès lors associer un comportement à une apparence (Rushen et al 1999). Cependant la réponse à un homme inconnu est fortement influencée par les précédents trai- tements donnés par les hommes familiers, démontrant la capacité des animaux non seulement à discriminer mais aussi à généraliser le comportement d'un éleveur familier à un inconnu. Cela permet à un évaluateur extérieur d'obtenir une information pertinente sur la relation éleveur-animal. Au final, il apparaît donc que les réactions des animaux lors des tests traduisent bien les interactions passées entre les animaux et l'homme ainsi que la perception de l'homme par l'animal et la qualité de la relation homme-animal.

L'application de l'éthologie pour développer des outils d'évaluation du comportement et du bien-être de l'animal ont permis de progresser dans la connaissance des facteurs affectant la réaction des animaux d'élevage à l'homme.

\section{3 / L'homme : une partie intégrante de l'univers relation- nel de l'animal}

Ces trente dernières années ont permis de mieux comprendre les facteurs et les mécanismes déterminant la perception de l'homme par l'animal. Des nombreuses synthèses écrites sur ce sujet (Hemsworth et Coleman 1998, Rushen et al 1999, Boivin et al 2003, Hausberger et al 2008, Hemsworth et Boivin 2011), il ressort les éléments suivants.

L'animal d'élevage peut montrer des réactions intenses de fuite voire d'attaque vis-à-vis de l'homme ou au contraire sembler rechercher son contact voire s'apaiser en sa présence. Comme pour tout comportement, trois grandes composantes ont été analysées : la composante génétique, la composante expérientielle (expérience vécue) et la composante de maturation liée à l'influence de l'environnement pendant le développement de l'individu. Il en ressort que les réactions de peur ont souvent pour origine une absence d'habituation à l'homme ou bien une peur apprise en relation avec des interventions négatives de l'homme en présence de l'animal (coups, cris, interventions douloureuses mais aussi mouvements d'agitation, courses, bruits effrayants...). A l'inverse, des contacts humains (alimentation, contacts tactiles, voix douces, mouvements calmes...) peuvent être perçus de façon positive par les animaux. Une part génétique très significative dans l'existence de ces réactions a également été montrée ; part relativement facile à démontrer chez les animaux d'élevage compte tenu du nombre souvent important de descendants par mâle reproducteur. Enfin, comme pour d'autres comportements, l'animal connaît au cours de sa vie des périodes 
Figure 1. Représentation schématique des différents facteurs impliqués dans la construction de la relation homme-animal.

La figure pointe l'importance des contacts humains positifs, neutres et négatifs et notamment la balance entre ceux-ci, qui doit être largement en faveur des premiers pour construire une relation homme-animal positive pour l'homme comme pour l'animal.
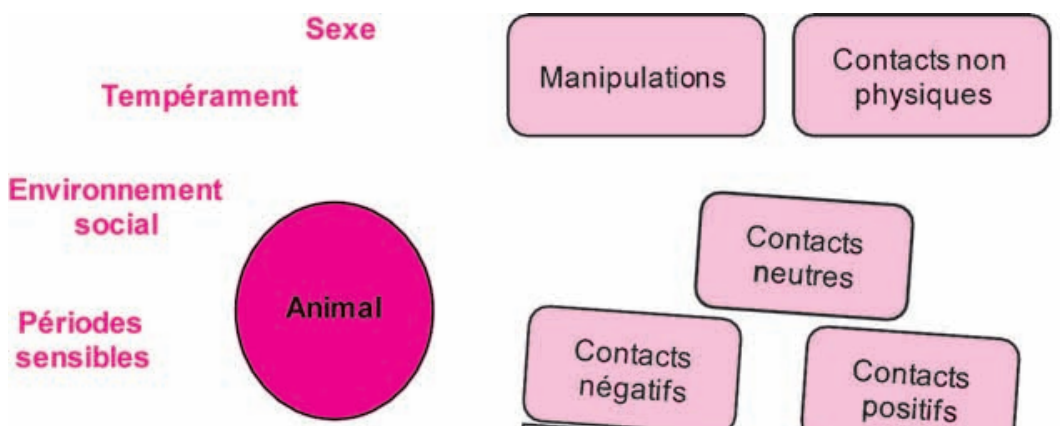

Systèmes d'élevage
Génétique

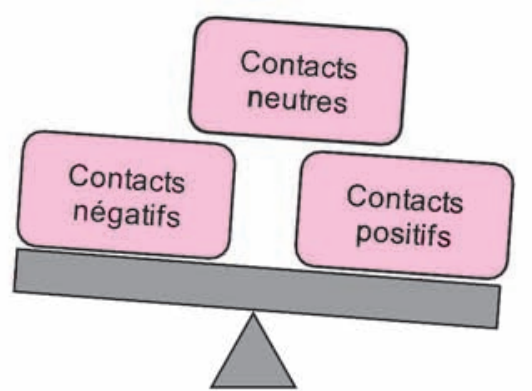

d'élevage pour étudier leur relation conduit à s'interroger sur l'existence de «pratiques relationnelles» en élevage. Au niveau de l'éleveur, sa perception de l'animal et de son travail, sa façon de s'organiser, ses habitudes de travail vont avoir un impact sur sa façon de se comporter et donc sur sa relation avec l'animal. Au niveau de l'animal, il existe des facteurs internes propres à chaque individu, des facteurs d'environnement physique et social et des facteurs liés aux modalités et aux mécanismes d'actions des interactions entre l'homme et l'animal (attachement, familiarisation, renforcements positifs ou négatifs, habituation à la présence et aux contacts). Se fondant sur les théories classiques de l'apprentissage (renforcements positifs/négatifs des comportements d'approche ou de fuite des animaux), de nombreuses études (cf. revue de Hemsworth et Boivin 2011) suggèrent que la balance entre les contacts positifs et négatifs doit être largement en faveur des premiers (figure 1) pour construire une relation positive avec l'animal,

\section{Représentation du métier \\ Représentation de l'animal

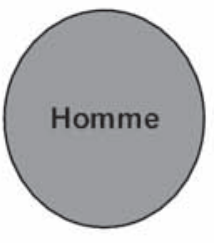 \\ Psychologie \\ Habitudes du travail}

c'est-à-dire dans laquelle la présence de l'homme et les manipulations ne sont pas aversives pour l'animal, mais peuvent même constituer une source d'enrichissement ou d'apaisement.

A l'heure actuelle, développer les contacts humains positifs apparaît d'autant plus important que le temps passé auprès des animaux et le nombre d'interactions ont tendance à se restreindre aux interactions d'élevage nécessaires par exemple aux soins, interactions souvent perçues comme négatives par l'animal (Hemsworth et Boivin 2011). Un modèle simple (figure 2) a été construit à partir de ces recherches suggérant que le comportement de l'éleveur peut générer une peur apprise de l'animal vis-à-vis de l'homme, un stress chronique chez l'animal en présence de l'éleveur qui peut impacter sa production, sa santé et sa reproduction (Hemsworth et Coleman 1998). Nous avons déjà mentionné le fait que les habitudes comportementales de l'éleveur dépendent de ses croyances sur s'expliquent non seulement du fait différence de contact homme-animal suivant le mode d'élevage mais aussi du fait que le jeune animal est fortement focalisé sur la présence de la mère et son comportement envers le partenaire humain.

En conclusion, les différents résultats obtenus montrent que l'homme s'intègre effectivement dans l'univers relationnel des animaux.

\section{3 / De l'apport de connais- sances sur la relation homme-animal vers l'iden- tification des pratiques relationnelles}

La prise en compte simultanée des perceptions de l'éleveur et de l'animal

Figure 2. Représentation schématique simplifiée du fonctionnement de l'homme envers l'animal et des conséquences sur l'état émotionnel, le bien-être et la productivité de ce dernier.

En retour, le comportement de l'animal peut affecter les croyances de l'éleveur et son comportement envers l'animal selon un «cercle vicieux ou vertueux».

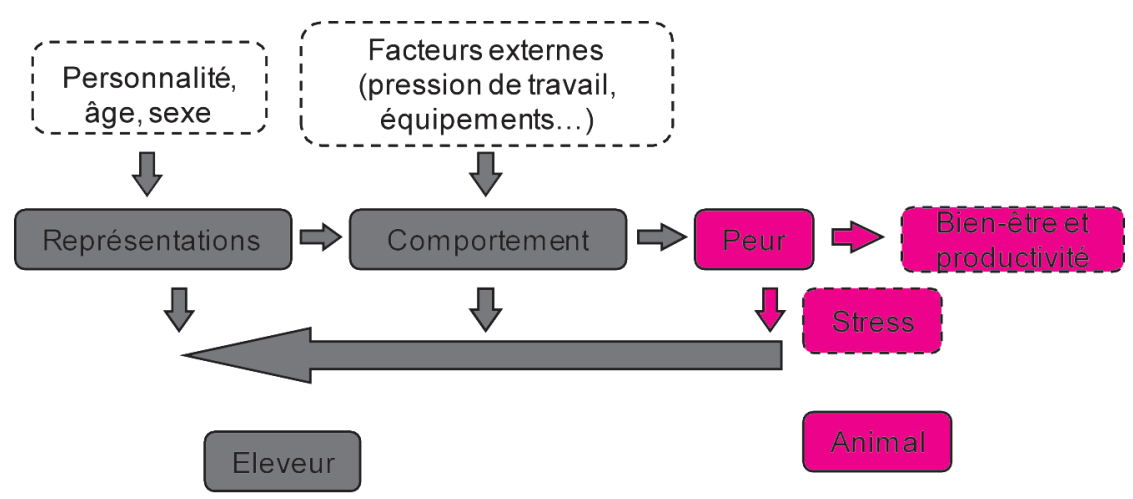


l'effet de ses actions sur le comportement de l'animal. Par exemple, un éleveur peut penser qu'un animal peureux demandera plus d'efforts, plus d'interventions négatives (coups, cris) qu'un animal calme. Il peut aussi croire qu'il ne sert à rien de toucher les animaux ou de leur parler calmement. Ses convictions sont issues de son histoire personnelle (par exemple ses premières expériences de manipulation) en relation avec sa personnalité, son âge, son sexe. Les études montrent que ces croyances «comportementales» sont des bons prédicteurs du comportement des personnes (Hemsworth et Coleman 1998). En retour, les réponses comportementales des animaux renforcent ces croyances. Plus un homme utilise des comportements aversifs envers l'animal pour le manipuler, plus ce dernier a peur, obligeant le manipulateur à davantage d'efforts pour arriver à ses fins. Cela a donc pour effet de renforcer ses convictions sur le comportement à adopter avec l'animal. Les facteurs externes tels que la pression de travail ou la mauvaise qualité des équipements empêchent souvent le manipulateur de réfléchir à d'autres façons de faire et aux conséquences de ses actions sur l'animal.

Pour lutter contre ces croyances qui génèrent des habitudes comportementales, il faut alors étudier ou concevoir des pratiques visant à améliorer la manipulation (moins de stress pour l'animal, confort et sécurité de l'homme et efficacité du travail) et le bien-être des animaux. On pourrait qualifier ses pratiques de «relationnelles» en référence à la définition de pratiques «zootechniques». Ces dernières peuvent s'envisager sous l'angle décisionnel, c'est-àdire par référence à un centre de décision (l'éleveur), aux ressources et à l'information dont il dispose, aux objectifs qu'il poursuit, aux stratégies qu'il développe à cet effet (Landais et al 1986). Les pratiques relationnelles pourraient inclure le choix de reproducteurs favorisant la réaction positive de leurs descendants face à l'homme, l'utilisation des périodes sensibles (jeune âge, sevrage..), l'optimisation des interactions positives, etc. On peut émettre l'hypothèse que, sans augmenter fortement le temps de travail, les résultats obtenus après formation des éleveurs pourraient très facilement et considérablement, améliorer la manipulation/ contention et le bien-être des animaux, ainsi que la qualité des relations homme-animal.

Afin de changer les représentations que l'homme a de l'animal et donc ses habitudes comportementales il est possible d'utiliser les techniques des Thérapies Cognitives et Comportementales (TCC). Les TCC cherchent essen- tiellement à modifier nos comportements indésirables en engageant une réflexion (cognition) sur la croyance que l'on a dans l'effet de nos comportements et en activant sur le terrain de nouveaux comportements plus adéquats par rapport aux résultats recherchés. Appliquant ces techniques lors de sessions de formation, Hemsworth et Coleman (1998) ont montré que les éleveurs peuvent faire évoluer leurs façons de faire et leurs habitudes de travail avec l'animal. Ces travaux ont apporté beaucoup pour améliorer concrètement la relation homme-animal en élevage porcin et bovin laitier. Ils ont été repris dans un programme de formation européen intitulé "Quality Handling» ${ }^{\circledR}$ en élevages de porcs, de volailles et de bovins. Mais ces formations s'intéressent pour l'instant aux interactions routinières entre l'éleveur et ses animaux. Une formation permettant aux éleveurs de réfléchir à leurs pratiques relationnelles intégrées dans leur organisation du travail pourrait permettre encore plus efficacement d'améliorer leur comportement face à l'animal.

\section{Conclusion}

De cette synthèse, il ressort qu'évaluer et améliorer la relation hommeanimal d'élevage au travers de la formation des éleveurs est bien un travail pluridisciplinaire, mais que de telles études ou formations sont rares. Le rapprochement entre sciences biotechniques et sciences sociales n'est pas facile. Nous constatons ce que l'on pourrait appeler un choc de culture lié à des problèmes épistémologiques. La démarche que les éthologues conduisent sur le comportement animal et la relation homme-animal peut apparaître comme trop réductrice de la complexité des rapports homme-animal («parcellisation» du comportement, Burgat 2010). Il peut exister aussi des difficultés pour concevoir des paradigmes communs qui satisfassent les standards quantitatifs et statistiques exigés par les sciences biotechniques et les approches souvent qualitatives des sciences humaines. L'approche de la relation homme-animal par l'analyse des pratiques relationnelles (sous l'angle décisionnel de la part de l'éleveur) pourrait permettre ce travail en commun entre sciences sociales et éthologie. L'observation directe des interactions homme-animal, et leur analyse à différents niveaux (origines, conséquences) peuvent fédérer les approches et sont essentielles pour corroborer paroles d'acteurs, travail réel et ressenti des animaux. Chaque discipline pourra apporter aux autres sa perspective, sa méthodologie, soit du côté de l'animal, soit du côté de l'homme. Une fois ces approches confrontées en grou- pe de travail, la réflexion pourra se poursuivre sur le terrain en menant une analyse pluridisciplinaire de la relation homme-animal dans les élevages, allant jusqu'à proposer des approches pour le conseil aux éleveurs ou la formation. L'observation des interactions directes homme-animal se heurtera bien sûr aux difficultés de moyens (temps, déplacement, présence perturbante de l'observateur), cycle de vie des animaux (exemple des périodes de vie variées chez les éleveurs naisseurs : jeune âge, sevrage, gestation, mise bas) et à la nécessité de faire des choix. Mais ces difficultés ne sont pas forcément insurmontables.

Afin de mieux comprendre les facteurs impliqués dans la perception et les réactions de l'animal à l'homme, et afin de construire si besoin de nouvelles pratiques relationnelles prenant en compte, et le point de vue des hommes et le point de vue des animaux, les questions suivantes sont à aborder de façon pluridisciplinaire

- Quelles sont les pratiques relationnelles majoritaires ou émergentes ?

- Dans quelle mesure, les éleveurs/ intervenants prennent ou non en compte les capacités émotionnelles et cognitives des animaux (réelles ou supposées) dans leur représentation du travail avec l'animal et dans leur travail réel (organisation générale du travail ou par poste).

- Quels facteurs biologiques entrent en compte pour les éleveurs dans leurs pratiques relationnelles (type d'animal, taille du troupeau, périodes sensibles tels le jeune âge, le sevrage ou la mise bas, environnement social...)?

- Quelles sont les conséquences réelles de ces pratiques en termes de production, de satisfaction et de sécurité au travail, ainsi qu'en termes de bien-être animal ? On peut enfin s'interroger sur la façon dont les éleveurs les intègrent dans l'organisation générale du travail (organisation du temps et de la qualité des contacts, activités leviers/pivots...).

Un large et passionnant champ de recherche s'ouvre sur ces questions.

\section{Remerciements}

Les rencontres ayant servi de base à la rédaction de cet article ont été financées par le projet CASDAR 9111 «Evaluation du Bien-être animal», avec la contribution financière du compte d'affectation spécial du développement agricole et rural, MAAPRAT. Sandy Bensoussan a participé à ce travail dans le cadre de son mémoire d'ingénieur agronome à Bordeaux Sciences Agro en 2010 . 


\section{Références}

Anonyme, 2006. Caractérisation des systèmes de production cunicole français et perspectives d'avenir. Cuniculture Magazine, 33, 78-85

Boivin X., Le Neindre P., Chupin J.M., Garel J.P., Trillat G., 1992. Influence of breed and early management on ease of handling and open-field behaviour of cattle. Appl. Anim. Behav. Sci., 32, 313-323.

Boivin X., Lensink J., Tallet C., Veissier I., 2003. Stockmanship and farm animal welfare. Anim. Welf., 12, 479-492.

Boivin X., Marcantognini L., Boulesteix P., Godet J., Brule A., Veissier I., 2007. Attitudes of farmers towards Limousin cattle and their handling. Anim. Welf., 16, 147-151.

Boivin X., Gilard F., Egal D., 2009. The effect of early human contact and the separation method from the dam on responses of beef calves to humans. Appl. Anim. Behav. Sci., 120, 132-139.

Bokkers E.A.M., Leruste H., Heutinck L.F.M., van der Werf J.T.N., Lensink B.J., Van Reenen C.G., 2009. Inter-observer and testretest reliability of on-farm behavioural observations in veal calves. Anim. Welf., 18, 381390.

Burgat F., 2010. Pensez le comportement : aux fondements des options epistemologiques. Burgat F. (Ed). Pensez le comportement animal : contribution à une critique du réductionnisme. Editions de la Maison des sciences de l'homme. Editions Quae, Paris, France, 17-49.

Campan R., Scapini F., 2002. Ethologie: Approche systémique du comportement. De Boeck Université, Bruxelles, Belgique, 737p.

Chauvat S., Servière G., 2005. «Améliorer les conditions de travail en élevage ovin viande pour lever un frein majeur à son développement.» Compte-rendu 1100550014. Institut de l'Elevage/Département Actions régionales, Juin 2005, 9p.

Cournut S., Chauvat S., 2012. L'organisation du travail en exploitation d'élevage : analyse de 630 Bilans Travail réalisés dans huit filières animales. In : Numéro spécial, Travail en élevage. Hostiou N., Dedieu B., Baumont R. (Eds). INRA Prod. Anim., 25, 101-112.

Darses F., de Montmollin M., 2006. L'ergonomie. La Découverte, Collection Repères, Paris, France, 43, 128p.

Dedieu B., Servière G., 1999. Caractériser et évaluer l'organisation du travail en élevage. La méthode Bilan Travail. Fasade, 1, 4p.

Dockes A.C., Kling-Eveillard F., 2007. Les représentations de l'animal et du bien-être animal par les éleveurs français. In : Numéro spécial, Bien-être animal. INRA Prod. Anim., 20, 23-28.

Dufour A., Dedieu B., 2010. Le travail en élevage et ses transformations : analyses sociologiques. Le travail en agriculture dans les sciences pour l'action. Journées d'étude INRA Sad-Cirad ES, Parent, 11p.

English P.R., 1991. Stockmanship, empathy and pig behaviour. The Pig Vet. J., 26,56-66.

Estep D.Q., Hetts S., 1992. Interactions, relationships, and bonds: the conceptual basis for scientist-animal relations. In: The inevitable bond: Examining scientist-animal interactions.
Davis H., Balfour D. (Eds), Cambridge University Press, Cambridge, UK, 6-26.

Fiorelli C., 2010. L'aménagement des conditions de vie au travail des éleveurs. Proposition d'un cadre d'analyse des relations entre rapport subjectif et organisation du travail en élevage et étude de cas chez les éleveurs pluriactifs. Thèse de doctorat, AgroParisTech., 312p

Fiorelli C., Mouret S., Porcher J., 2012. Les rationalités du travail avec les animaux d'élevage : produire, vivre ensemble et se construire. In : Numéro spécial, Travail en élevage. Hostiou N., Dedieu B., Baumont R. (Eds). INRA Prod. Anim., 25, 181-192.

Fishbein M., 1980. A theory of reasoned action: some applications and implications. In: H.E. Howe Jr., M.M. Page (Eds). Nebraska Symposium on Motivation, Lincoln: University of Nebraska Press, USA, 27, 65-116.

Gaudillère N., 2009. Temps de travail enquêtes dans 59 élevages laitiers. Terra, 38, 1

Grannec M.L., 2010. Référentiel travail. Synthèse détaillée filière porcine. Chambres d'Agriculture de Bretagne et des Pays de la Loire, 20p.

Hausberger M., Roche H., Henry S., Visser E.K., 2008. A review of the human-horse relationship. Appl. Anim. Behav. Sci., 109, 1-24

Hemsworth P, Boivin X., 2011. Human Contact. In: Animal Welfare (2nd edition). M.C. Appleby J.A. Mench I.A.S. Olsson B.O. Hughes (Eds), CAB International, Cambridge, UK, 246-262.

Hemsworth P.H., Coleman G.J., 1998. Human-Livestock interactions: the stockperson and the productivity and welfare of intensively farmed animals. CAB International, 152p.

Hemsworth P.H., Barnett J.L., Hansen C., 1986. The influence of handling by humans on the behaviour, reproduction and corticosteroids of male and female pigs. Appl. Anim. Behav. Sci., 15, 303-314.

Hemsworth P.H., Barnett J.L., Hansen C. 1987. The influence of inconsistent handling by humans on the behaviour, growth and corticosteroids of young pigs. Appl. Anim. Behav. Sci., 17, 245-252.

Hemsworth P.H., Barnett J.L., Coleman G.J., Hansen C., 1989. A study of the relationships between the attitudinal and behavioural profiles of stockpersons and the level of fear of humans and reproductive performance of commercial pigs. Appl. Anim. Behav. Sci., 23, $301-$ 314.

Hemsworth P.H., Barnett J.L., Treacy D., Madgwick P., 1990. The heritability of the trai fear of humans and the association between this trait and subsequent reproductive performance of gilts. Appl. Anim. Behav. Sci, 25, 85-95.

Hemsworth P.H., Coleman G.J., Barnett J.L. Jones R.B., 1994a. Behavioural responses to humans and the productivity of commercia broiler chickens. Appl. Anim. Behav. Sci., 41, 101-114.

Hemsworth P.H., Coleman G.J., Barnett J.L., 1994b. Improving the attitude and behaviour of stockpersons toward pigs and the consequences on the behaviour and reproductive performance of commercial pigs. Appl. Anim. Behav. Sci. 39, 349-362.
Hemsworth P.H. Coleman G.J., Barnett J.L, Borg S., 2000. Relationships between humananimal interactions and productivity of commercial dairy cows. J. Anim. Sci., 78, 28212831 .

Jones R.B., 1993. Reduction of the domestic chick's fear of human beings by regular handling and related elements. Anim. Behav. 46, 991-998.

Kling-Eveillard F., Dockès A.C., Mirabito L., Capdeville J., Magdelaine P., Courboulay V., 2009. Le bien-être animal dans les démarches qualité : point de vue des éleveurs. Editions Institut de l'élevage, Paris, France, 8p.

L'Hotellier N., Dessors D., Flottes A., 2007. De la relation avec l'animal à la formation au travail avec ce dernier : Quelles difficultés ? Quels enjeux ? Rapport Final

Lamine C., 2006. Mettre en parole les relations entre hommes et animaux d'élevage. Circulation des récits et mise en débat. http://www.ethnographiques.org/2006/Lamine. $\underline{\text { html }}$

Landais E., Lhoste P., Milleville P., 1986. Points de vue sur la zootechnie et les systèmes d'élevage tropicaux. Cah. Sci. Hum., 23, 421 437.

Le Guen R., 2006. La diversité des logiques de travail en production laitière. Fourrages. 185, 25-34.

Lensink B.J., Boissy A., Veissier I., 2000. The relationship between farmers' attitude and behaviour towards calves, and productivity of veal units. Appl. Anim. Behav. Sci., 49, 313327.

Metz J.H.M., 1986. The response of farm animals to humans - Examples of the epistemology of experimental research. In: Proceedings of a seminar in the community programme for coordination of agricultural research. Bruxelles, Belgique, 22-37.

Montmeas L., Ourliac S., Prodhomme J., Pucelle, L., 2006. Manipulation et intervention sur le bovins, Educagri Editions, Dijon, France, $184 \mathrm{p}$

Porcher J., 1997. La relation de communication entre l'éleveur et ses animaux: un domaine encore à explorer. Le courrier de l'environnement, 32, 51-62.

Porcher J., 2001. L'élevage, un partage de sens entre hommes et animaux : intersubjectivité des relations entre éleveurs et animaux dans le travail. Thèse de doctorat, Institut National Agronomique Paris-Grignon, France, 205 p.

Procoli A., 2004. Le temps et la construction du regard sur l'animal de rente. Ethnographie des pratiques et récits des éleveurs bretons. Cah. Econ. Sociol. Rurales, 72, 92-113.

Renger H., 1975. Agressive Verhalten von Bullen dem Menschen gegenüber. Diss Med.Vet., LMU München, Allemagne, 80p.

Rushen J., Taylor A.A., de Passillé A.M., 1999. Domestics' animals fear of humans and its effects on their welfare. Appl. Anim. Behav. Sci., 65, 285-303.

Seabrook M.F., 1972. A study to determine the influence of the herdsman personality on milk yield. J. Agric. Labour Sci., 1, 45-59. 
Veissier I., Botreau R., Perny P., 2010. Evaluation multicritère appliquée au bien-être des animaux en ferme ou à l'abattoir : difficultés et solutions du projet Welfare Quality ${ }^{\circledR}$. INRA Prod. Anim., 23, 269-284.
Waiblinger S., Menke C., Folsch D.W. 2003. Influences on the avoidance and approach behaviour of dairy cows towards humans on 35 farms. Appl. Anim. Behav. Sci., 84, 23-39
Waiblinger S., Boivin X., Pedersen V., Tosi M.V., Janczak A.M., Visser E.K., Jones R.B., 2006. Assessing the human-animal relationship in farmed species: A critical review. Appl. Anim. Behav. Sci., 101, 185-242.

\section{Résumé}

Le statut juridique de l'animal reconnu comme «être sensible», induit de fait qu'une réflexion sur le travail en élevage et la relation homme-animal doit prendre en compte le point de vue de l'éleveur, et celui de l'animal. Les enjeux sociétaux, se situent autant en termes d'efficacité du travail et de qualité de vie pour les professions agricoles, qu'en terme de bien-être animal. Cet article de synthèse s'intéresse à la pluridisciplinarité, en particulier entre sciences sociales et éthologie afin de comprendre et d'améliorer les pratiques d'élevage déterminantes dans la relation homme-animal. L'analyse du temps de travail, regardée sous l'angle des contacts hommeanimal révèle une forte diversité suivant les filières et les exploitations. Les diversités de profils des éleveurs, de leurs représentations de l'animal et du travail avec l'animal, et de leurs logiques d'élevage sont rapportées. De son côté, l'éthologie définit un cadre conceptuel fondé sur le fait que l'éleveur et l'animal construisent par leurs interactions répétées une relation interindividuelle qui leur permet de prévoir l'issue de leurs futures interactions. La réaction des animaux à l'homme traduit donc une synthèse des interactions passées et permet d'une certaine façon d'évaluer leur relation. Les études en éthologie montrent que l'animal intègre effectivement l'homme dans son univers relationnel. L'article pointe l'intérêt de décrire la variabilité des pratiques relationnelles de l'éleveur et de les évaluer afin d'obtenir une relation homme-animal bénéfique tant pour l'homme que pour l'animal.

\section{Abstract \\ Humans and livestock animals at work: toward a multidisciplinary approach of relational practices}

The legal status of the animal defined as a "sentient being" involves taking into account both the breeder's and animals' points of view for considering work in livestock productions and the human-animal relationship. Farmers' work efficiency, quality of life and animal welfare are targeted. This review paper focuses on a multidisciplinary approach, in particular between the social sciences and ethology in order to understand and improve husbandry practices critically affecting the human-animal relationship. On the one hand, a working time analysis focused on the human-animal contact, reveals a strong diversity among production systems and farms. The diversity in breeders' profiles, attitudes towards animals and their work with animals and in their husbandry strategies are reported. On the other hand, ethology elaborates a conceptual framework based on the fact that farmers and animals build their relationship from the repeated interactions they display. This relationship allows them to predict the issue of the future interactions. Observing the animal's responses to the human represents a synthetic evaluation tool for evaluating their relationship. Ethological studies showed that humans integrate the relational world of animals. This paper points out the interest of describing and evaluating the existing farmers' relational practices towards animals, and developing new and beneficial ones both for farmers and animals.

BOIVIN X., BENSOUSSAN S., L'HOTELLIER N., BIGNON L., BRIVES H., BRULE A., GODET J., GRANNEC M.-L., HAUSBERGER M., KLING-EVEILLARD F., TALLET C., COURBOULAY V., 2012. Hommes et animaux d'élevage au travail : vers une approche pluridisciplinaire des pratiques relationnelles. In : Numéro spécial, Travail en élevage. Hostiou N., Dedieu B., Baumont R. (Eds). INRA Prod. Anim., 25, 159-168. 